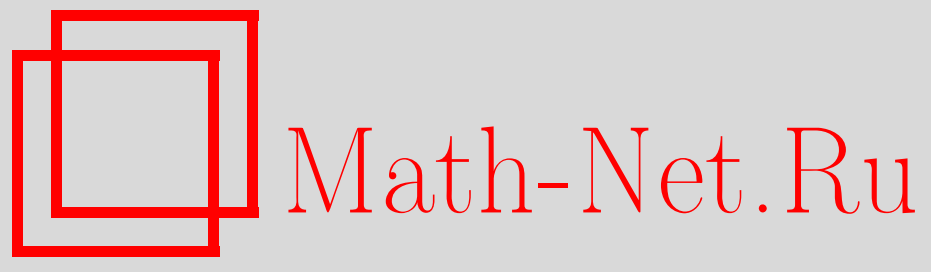

В. В. Горгорова, И. В. Павлов, О свойствах хааровской единственности для векторнозначных случайных процессов, УМH, 2007, том 62, выпуск 6, 169-170

DOI: https://doi.org/10.4213/rm8557

Использование Общероссийского математического портала Math-Net.Ru подразумевает, что вы прочитали и согласны с пользовательским соглашением http://www.mathnet.ru/rus/agreement

Параметры загрузки:

IP: 3.91 .87 .62

26 апреля 2023 г., $11: 40: 37$

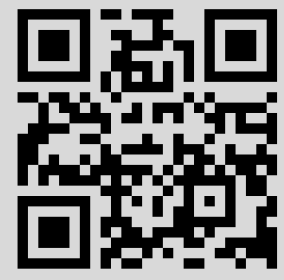




\section{О свойствах хааровской единственности для векторнозначных случайных процессов}

\section{В. В. Горгорова, И. В. Павлов}

Рассмотрим фильтрованное пространство (ф.п.) $\left(\Omega, \mathbb{F}=\left(\mathscr{F}_{k}\right)_{k=0}^{N}, \mathscr{F}\right)$, где число $N$ конечно, все $\sigma$-алгебры $\mathscr{F}_{k}$ конечны, $\mathscr{F}_{0}=\{\Omega, \varnothing\}, \mathscr{F}=\mathscr{F}_{N}$ и $\mathscr{F}_{k} \neq \mathscr{F}_{k+1}$ при любом $k$. Обозначим через $\mathscr{P}$ множество вероятностных мер на $(\Omega, \mathscr{F})$, каждая из которых нагружает все атомы из $\mathscr{F}$. Пусть $Z=\left(Z_{k}, \mathscr{F}_{k}\right)_{k=0}^{N}$ - адаптированный векторнозначный случайный процесс (в.с.п.) на $(\Omega, \mathscr{F})$, где $Z_{k}=\left(Z_{k}^{(1)}, \ldots, Z_{k}^{(l)}\right)$. Как обычно, символ $\left.f\right|_{A}$ означает ограничение случайной величины (с.в.) $f$ на событие $A \in \mathscr{F}$. Пусть

$$
A=B_{1}+\cdots+B_{m},
$$

где $A$ - атом в $\mathscr{F}_{k}, B_{i}$ - атом в $\mathscr{F}_{k+1}(i=1, \ldots, m)$ и $1 \leqslant m<\infty(m$ зависит от $A$; это число мы будем называть характеристикой дробления атома $A \in \mathscr{F}_{k}$ ). Обозначим через $\mathscr{P}(Z, \mathbb{F})$ (соответственно $\mathscr{P}\left(Z^{(i)}, \mathbb{F}\right)$ ) множество мер $\mathrm{P}$ из $\mathscr{P}$, относительно которых в.с.п. $Z=\left(Z_{k}, \mathscr{F}_{k}, \mathrm{P}\right)_{k=0}^{N}\left(\right.$ соответственно процесс $\left.Z^{(i)}=\left(Z_{k}^{(i)}, \mathscr{F}_{k}^{(i)}, \mathrm{P}\right)_{k=0}^{N}\right)$ является мартингалом. Имеем: $\mathscr{P}(Z, \mathbb{F})=\bigcap_{i=1}^{l} \mathscr{P}\left(Z^{(i)}, \mathbb{F}\right)$.

Фильтрация $\mathbb{H}=\left(\mathscr{H}_{n}\right)_{n=0}^{L}$ на $(\Omega, \mathscr{F})$ называется хааровской, если для любого $n$ $(0 \leqslant n \leqslant L) \sigma$-алгебра $\mathscr{H}_{n}$ порождена разбиением $\Omega$ ровно на $n+1$ атом. Хааровская фильтрация $\mathbb{H}=\left(\mathscr{H}_{n}\right)_{n=0}^{L}$ называется интерполирующей хааровской фильтрацией (и.х.ф.) исходной фильтрации $\mathbb{F}$, если существует последовательность натуральных чисел $0=n_{0}<n_{1}<\cdots<n_{N}=L$, для которой $\mathscr{H}_{n_{k}}=\mathscr{F}_{k}$.

Предположим, что $\mathscr{P}(Z, \mathbb{F}) \neq \varnothing$, и зафиксируем меру $\mathrm{P} \in \mathscr{P}(Z, \mathbb{F})$. По мартингалу $Z=\left(Z_{k}, \mathscr{F}_{k}, \mathrm{P}\right)_{k=0}^{N}$ построим мартингальную хааровскую интерполяцию $Y=$ $\left(Y_{n}, \mathscr{H}_{n}\right)_{n=0}^{L}$, используя формулу $Y_{n}=\mathrm{E}^{\mathrm{P}}\left[Z_{N} \mid \mathscr{H}_{n}\right]$ (вероятностное решение задачи Дирихле). Если и.х.ф. $\mathbb{H}$ фильтрации $\mathbb{F}$ фиксирована, то процесс $Y$ определяется по мартингалу $Z$ однозначно.

Пусть $|M|$ обозначает число элементов множества $M$. Говорят, что мартингальная мера $\mathrm{P} \in \mathscr{P}(Z, \mathbb{F})$ удовлетворяет свойству хааровской единственности $(\mathrm{P} \in \operatorname{HUP}(Z))$, если для исходной фильтрации $\mathbb{F}$ можно построить такую и.х.ф. $\mathbb{H}$, что для соответствующей мартингальной интерполяции $Y=\left(Y_{n}, \mathscr{H}_{n}\right)_{n=0}^{L}$ имеет место соотношение $|\mathscr{P}(Y, \mathbb{H})|=1$ (т. е. только относительно исходной меры $\mathrm{P}$ процесс $Y$ является мартингалом). Более важным является следующее определение. Говорят, что мартингальная мера $\mathrm{P} \in \mathscr{P}(Z, \mathbb{F})$ удовлетворяет свойству универсальной хааровской единственности $(\mathrm{P} \in \operatorname{UHUP}(Z))$, если для любой и.х.ф. $\mathbb{H}$ исходной фильтрации $\mathbb{F}$ имеет место соотношение $|\mathscr{P}(Y, \mathbb{H})|=1$, где $Y=\left(Y_{n}, \mathscr{H}_{n}\right)_{n=0}^{L}$ - соответствующая мартингальная интерполяция процесса $Z$. Аналогично определяются включения $\mathrm{P} \in \operatorname{HUP}\left(Z^{(i)}\right)$ и $\mathrm{P} \in \operatorname{UHUP}\left(Z^{(i)}\right)$. Ясно, что для любого $i(0 \leqslant i \leqslant l)$ справедливо $\mathscr{P}(Z, \mathbb{F}) \cap \operatorname{HUP}\left(Z^{(i)}\right) \subset \operatorname{HUP}(Z)$ и $\mathscr{P}(Z, \mathbb{F}) \cap \operatorname{UHUP}\left(Z^{(i)}\right) \subset \operatorname{UHUP}(Z)$. Из результатов работы [1] вытекает, что если $\operatorname{HUP}\left(Z^{(i)}\right) \neq \varnothing\left(\right.$ соответственно $\operatorname{UHUP}\left(Z^{(i)}\right) \neq \varnothing$ и для любого $k(0 \leqslant k<N)$ и любого атома $A \in \mathscr{F}_{k}$ характеристика дробления $m$ не превосходит 3), то $\operatorname{HUP}\left(Z^{(i)}\right)=\mathscr{P}\left(Z^{(i)}, \mathbb{F}\right)\left(\right.$ соответственно $\left.\operatorname{UHUP}\left(Z^{(i)}\right)=\mathscr{P}\left(Z^{(i)}, \mathbb{F}\right)\right)$. Отсюда вытекает справедливость следующей леммы.

Лемма 1. Если $\operatorname{HUP}\left(Z^{(i)}\right) \neq \varnothing\left(\right.$ соответственно $\operatorname{UHUP}\left(Z^{(i)}\right) \neq \varnothing$ и для любого $k$ $(0 \leqslant k<N)$ и любого атома $A \in \mathscr{F}_{k}$ характеристика дробления $m$ не превосходит 3$)$, то $\operatorname{HUP}(Z)=\mathscr{P}(Z, \mathbb{F})($ соответственно $\operatorname{UHUP}(Z)=\mathscr{P}(Z, \mathbb{F}))$.

На самом деле справедлива следующая теорема.

Tеорема 1. 1) Если $\operatorname{HUP}(Z) \neq \varnothing$, то для любого $k(0 \leqslant k<N)$ и любого атома $A \in \mathscr{F}_{k}$ с характеристикой дробления $m$, строго большей 1 , существует номер $i$ 
такой, что выполняется неравенство

$$
\left.\min _{1 \leqslant j \leqslant m} Z_{k+1}^{(i)}\right|_{B_{j}}<\left.Z_{k}^{(i)}\right|_{A}<\left.\max _{1 \leqslant j \leqslant m} Z_{k+1}^{(i)}\right|_{B_{j}} .
$$

2) Если для любого $k(0 \leqslant k<N)$ и любого атома $A \in \mathscr{F}_{k}$ с характеристикой дробления $m$, строго большей 1 , существует номер $i$ такой, что выполняется неравенство (1), то $\operatorname{HUP}(Z)=\mathscr{P}(Z, \mathbb{F})$.

Данная теорема является обобщением на случай векторнозначных процессов соответствующего одномерного результата из [2].

СледствиЕ 1. Если $\mathbb{F}$ - естественная фильтрация процесса $Z$, то выполняется условие (1) и поэтому $\operatorname{HUP}(Z)=\mathscr{P}(Z, \mathbb{F})$.

ЗАмечАниЕ 1 . Сформулированная теорема 1 остается верной, если $l=\infty$, а также в случае, когда $\Omega$ счетно, а $l<\infty$ (определение в последнем случае интерполирующей хааровской фильтрации, а также обсуждение случая $l=1$ имеется в [2]).

Теорема 2. Пусть для любого $k(0 \leqslant k<N)$ и любого атома $A \in \mathscr{F}_{k}$ характеристика дробления $m$ не превосходит 3 и существует номер $i$ такой, что числа $\left.Z_{k}^{(i)}\right|_{A},\left.Z_{k+1}^{(i)}\right|_{B_{1}}, \ldots,\left.Z_{k+1}^{(i)}\right|_{B_{m}}$ различны. Тогда $\operatorname{UHUP}(Z)=\mathscr{P}(Z, \mathbb{F})$.

ЗАмечание 2. Утверждение, обратное теореме 2, неверно. Действительно, пусть $N=1, l=2$ и множество $\Omega$ дробится на три атома $\omega_{1}, \omega_{2}, \omega_{3}$. Пусть $Z_{0}^{(1)}=Z_{0}^{(2)}=2$, $Z_{1}^{(1)}\left(\omega_{1}\right)=4, Z_{1}^{(1)}\left(\omega_{2}\right)=2, Z_{1}^{(1)}\left(\omega_{3}\right)=1, Z_{1}^{(2)}\left(\omega_{1}\right)=1, Z_{1}^{(2)}\left(\omega_{2}\right)=4, Z_{1}^{(2)}\left(\omega_{3}\right)=2$. Легко видеть, что в данном случае $|\mathscr{P}(Z, \mathbb{F})|=1$, т. е. $\operatorname{UHUP}(Z)=\mathscr{P}(Z, \mathbb{F})$. Однако условия теоремы 2 не выполняются ни при $i=1$, ни при $i=2$. Таким образом, из того, что $\operatorname{UHUP}(Z) \neq \varnothing$, не следует существование такого номера $i$, что $\operatorname{UHUP}\left(Z^{(i)}\right) \neq \varnothing$.

Приведем также один результат, дающий конструкцию мартингальной меры из множества $\operatorname{UHUP}(Z)$, когда условия теоремы 2 не выполняются. Пусть $\left(\Omega^{(i)}\right.$, $\left.\left(\mathscr{F}_{k}^{(i)}\right)_{k=0}^{N}, \mathscr{F}^{(i)}\right)-$ ф.п. $(i=1,2)$, где $N<\infty, \mathscr{F}_{0}^{(i)}=\left\{\Omega^{(i)}, \varnothing\right\}$, а $\mathscr{F}^{(i)}=\mathscr{F}_{N}^{(i)}-$ конечные $\sigma$-алгебры. Обозначим через $\mathbb{F}^{(i)}$ фильтрацию $\left(\mathscr{F}_{k}^{(i)}\right)_{k=0}^{N}$. Рассмотрим $\Omega=$ $\Omega^{(1)} \times \Omega^{(2)}, \mathscr{F}_{k}=\mathscr{F}_{k}^{(1)} \otimes \mathscr{F}_{k}^{(2)}, \mathscr{F}=\mathscr{F}^{(1)} \otimes \mathscr{F}^{(2)}$ и обозначим $\mathbb{F}=\left(\mathscr{F}_{k}^{(1)} \otimes \mathscr{F}_{k}^{(2)}\right)_{k=0}^{N}$.

Tеорема 3. Пусть процесс $\left(Z_{k}^{(i)}, \mathscr{F}_{k}^{(i)}\right)_{k=0}^{N}$ допускает единственную мартингальную меру $\mathrm{P}^{(i)}$, нагружсаюшую все атомы $\sigma$-алгебры $\mathscr{F}^{(i)}(i=1,2)$. Тогда мера $\mathrm{P}=\mathrm{P}^{(1)} \otimes \mathrm{P}^{(2)}$ принадлежит множеству $\operatorname{UHUP}(Z)$.

\section{Список литературы}

[1] М. Н. Богачева, И. В. Павлов, УМН, 57:3 (2002), 143-144; англ. пер.: М. N. Bogacheva, I. V. Pavlov, Russian Math. Surveys, 57:3 (2002), 581-583. [2] И. В. Павлов, А. Г. Данекянц, Обозрение прикл. и промышл. матем., 11:1 (2004), 73-82.

\section{В. В. Горгорова (V. V. Gorgorova)}

Ростовский государственный строительный университет E-mail: gorgorovav@aaanet.ru

И. В. Павлов (I. V. Pavlov)

Ростовский государственный строительный университет

E-mail: pavloviv2005@mail.ru
Представлено Д. В. Трещёвым Принято редколлегией 08.10.2007 